TABLE I. Counter fillings used.

\begin{tabular}{lcc}
\hline & Counter $I$ & Counter $I I$ \\
\hline Xenon & $50 \mathrm{~cm} \mathrm{Hg}$ & $26 \mathrm{~cm} \mathrm{Hg}$ \\
Argon & $10 \mathrm{~cm} \mathrm{Hg}$ & $14 \mathrm{~cm} \mathrm{Hg}$ \\
Methane & $\sim 10 \mathrm{~cm} \mathrm{Hg}$ Hg & $10.2 \mathrm{~cm} \mathrm{Hg}$ \\
Hydrogen & $\sim 7000 \mathrm{counts} / \mathrm{min}$. & $\sim 30,000 \mathrm{counts} / \mathrm{min}$. \\
$\mathrm{H}^{3}$ & $\sim 6000$ counts $/ \mathrm{min}$. \\
$\mathrm{A}^{37}$ & & \\
\hline
\end{tabular}

Both counters were operated at gas multiplication factors of several thousand. The absolute energy scale was obtained by firing into the counter a beam of Mo $K \alpha$-x-rays $(17.4 \mathrm{kev})$ from a crystal spectrometer. In counter $I$ this beam was parallel to the counter wire, in $I I$ perpendicular to it. The assumption that these energy calibrations were representative of the properties of the counter as a whole was checked directly for counter $I I$ by measuring the Mo $K \alpha / \mathrm{A}^{37}$ pulse size ratio, ${ }^{*}$ and is inferred for counter $I$ from the agreement between the end point energy determinations in the two counters.

The complete spectrum was investigated in counter $I$. Since counter linearity had to be maintained up to $20 \mathrm{kev}$, we were not able to use multiplication factors as high as those used in the investigation ${ }^{4}$ of the $\mathrm{Cl} L_{I}$ peak $(280 \mathrm{ev})$. Consequently the amplifier noise was apparent at energies as high as about $600 \mathrm{ev}$.

At the ends of the counter the multiplication falls off due to reduced field strength. Disintegrations occurring in this region will produce pulses of spuriously low amplitude. Clearly the shape of the spectrum is most affected at low energy. Due to lack of data the correction to be applied is uncertain, a fact which precludes a quantitative comparison of our results with Fermi's theory in the region near the most probable energy $(\sim 2.5 \mathrm{kev})$. However the results agree with the theory within the limits of the correction uncertainty $(\sim 10$ percent at $1 \mathrm{kev})$.

Above $5 \mathrm{kev}$ the correction is very small, and close agreement with the theory is obtained (wall effects are negligible). We have concentrated on this energy region, in particular we have used both counters for a careful investigation of the end point. Using a biased amplifier, the spectrum above $15 \mathrm{kev}$ was expanded over the pulse analyzer. The results were compared with Fermi's theory, assuming a zero neutrino rest-mass. The theoretical distribution $N(\epsilon)$ can be written as constant $\times \epsilon^{2}\left(\epsilon_{0}-\epsilon\right)^{2} \theta(Z, \epsilon)$, where $\theta$ gives the Coulomb field effect. ${ }^{5}$ In counter $I$ the 17.4-kev calibration line was found to have a Gaussian shape of standard deviation $\sigma=700 \mathrm{ev}$, over a range of at least $\pm 3 \sigma$. In counter $I I \sigma$ was $1100 \mathrm{ev}$. The corrections to be applied for this line width were obtained by calculating the effect of a Gaussian spread on the theoretical distribution. A provisional value for the end point energy is adopted:** the corrected data are then used to redetermine the end point.

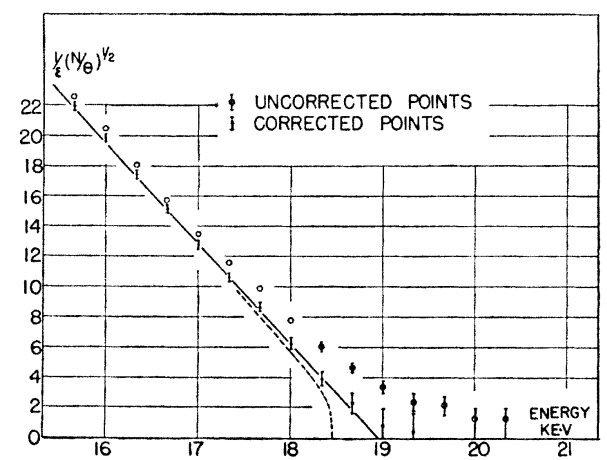

FIG. 2. "Kurie" plot of the end of the $\mathrm{H}^{3}$ spectrum. The theoretical curve (shown dotted) corresponding to a finite neutrino mass of $500 \mathrm{ev}$ (or $1 \mathrm{kev}$ (shown dotted) corresponding to a finite neutris
A theoretical curve based on this new value is then used as before. The progress of successive approximations is extremely rapid.

Figures 1 and 2 show the experimental and corrected points obtained using counter $I$. The fact that the corrected points lie on the assumed theoretical curve from which the corrections were computed means that our initial assumption of a zero neutrino mass is correct, within our limits of error.

The dotted curve in Fig. 2 has been drawn to show the effect of a neutrino mass of $500 \mathrm{ev}^{6}$ (or $1 \mathrm{kev}$ if a later modification $^{7}$ of the theory is accepted). If this distribution were used to correct for line width, a smaller correction would result and the discrepancy between the corrected data and the dotted curve would be even more marked than shown in Fig. 2. Nevertheless, in view of the width correction magnitude and the finite $(300 \mathrm{ev})$ pulse analyzer channel width, we do not feel justified in definitely excluding a finite value of $500 \mathrm{ev}$ (or $1 \mathrm{kev}$ ) for the neutrino mass.

Very similar results were obtained from the data of counter $I I$ : there is again no evidence for a neutrino mass greater than zero. The corrections for line width, however, are greater than for counter $I$.

The extrapolated end-point energy from the corrected data, is for counter $I, 18.95$, and for counter $I I, 18.9 \mathrm{kev}$. We believe that the true end point is at $18.9(5) \mathrm{kev}$ within $\pm 0.5 \mathrm{kev}$.

The first publication ${ }^{3}$ of Curran et al. gave a value of 16.9 $\pm 0.3 \mathrm{kev}$. This has recently ${ }^{8}$ been increased to $17.9 \pm 0.3 \mathrm{kev}$. Calibration lines ( $\mathrm{Cu}$ and $\mathrm{Ni} K \mathrm{x}$-rays) of energy about onehalf of the end-point energy were used.

1 D. H. W. Kirkwood, B. Pontecorvo, and G. C. Hanna, Phys. Rev. 74 497 (1948).

985 (1949)

${ }^{3} \mathrm{~S}$. C. Curran, J. Angus, and A. L. Cockcroft, Nature 162, 302 (1948).

${ }^{*} \mathrm{~A}^{37}$ gives a 2.8 -kev calibration line which is truly representative, since,
as for $\mathrm{H}^{3}$, the disintegrations occur uniformly throughout the counter volume.

'B. Pontecorvo, D. H. W. Kirkwood, and G. C. Hanna, Phys. Rev. 75 982 (1949).

5 E. Bleuler and W. Zünti, Helv. Phys. Acta 19, 375 (1946).

** Fortunately the correction is small for points lying more than $1.5 \sigma$ away from the end point so that a fairly good value of the end point is available immediately from the extrapolation of a "Kurie" plot (Fig. 2) o uncorrected data. For example in counter $I$ this value is greater by only $300 \mathrm{ev}$ than the one finally derived

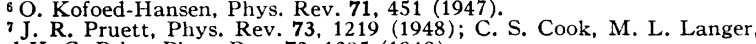
and H. C. Price, Phys. Rev. 73, 1395 (1948).

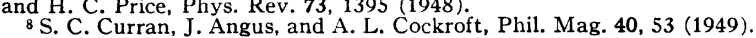

\section{Ferromagnetic Alloys in the System Copper-Manganese-Indium}

Robert R. GRINSTEAD AND Don M. Yost Gates and Crellin Laboratories of Chemistry. California Institute of Technology,

Pasadena, Californic

I $\mathrm{N}$ a recent publication by Hames and Eppelsheimer on ferromagnetic manganese alloys, ${ }^{1}$ reference was made to an article by $\mathrm{S}$. Valentiner, ${ }^{2}$ who has reported the existence of ferromagnetic alloys in the system $\mathrm{Cu}-\mathrm{Mn}$ - In, with a maximum intensity of magnetization at the point $\mathrm{Cu}_{2} \mathrm{MnIn}$. We have been working on this same system, and since the journal containing Valentiner's report is not available as yet, we wish to report some of the results of our work.

About fifteen alloys have been prepared, most of them containing a constant atomic ratio of $2 \mathrm{Cu}: 1 \mathrm{Mn}$ and with the indium content increasing from zero in steps of 5 atom percent to 60 atom percent, the latter being the most recent preparation. Except for the $\mathrm{Cu}-\mathrm{Mn}$ binary alloys all of the specimens prepared were definitely ferromagnetic. No quantitative measurements of intensity of magnetization have yet been made; the Curie point of an alloy composed of copper, manganese, and indium in the approximate atomic ratio of $45: 20: 35$ is about $250^{\circ} \mathrm{C}$ 
Our work has consisted mainly of thermal analyses, since the system had been previously unreported, and thorough knowledge of the phase relations was deemed necessary before proceeding to the measurement of magnetic and other properties. In most cases, two or three breaks were observed in the cooling curves during solidification; however, in one, namely, for the alloy of composition corresponding to $\mathrm{Cu}_{2} \mathrm{MnIn}$, only one break could be found. The plateau that followed was at about $617^{\circ} \mathrm{C}$ and probably indicates a compound or an eutetic mixture at this composition.

Further thermal measurements are in progress, and it is intended to supplement them with microscopic examinations of the properly heat-treated alloys. By means of these data, it should be possible to determine whether a single phase is responsible for the magnetism, and if so, to determine its composition and temperature limits. However, in view of the large range of composition which exhibits ferromagnetism, it would not be surprising to find more than one magnetic phase in this system.

1 Hames and Eppelsheimer, Nature 162, 968 (1948).

2 S. Valentiner, Naturwiss. 4, 123 (1947)

\section{Discharge of a Geiger Counter at Voltages above the Plateau}

\author{
H. R. CRANe \\ Randall Laboratory of Physics, University of Michigan \\ Ann Arbor, Michigan \\ January 17, 1949
}

W

$\mathrm{HEN}$ a voltage higher than that corresponding to the upper end of the plateau is applied to a Geiger counter, a discharge occurs which is spoken of as continuous, but which, in fact, is a very rapid succession of pulses. I have been interested in trying to learn something about the mechanism by which a counter first breaks into such a discharge and the mechanism by which succeeding pulses are produced. It is difficult to see how the existence of the electric field can, of itself, produce the first electron or ion in order to initiate the breakdown. The onset of field emission from the cathode surface surely would not be expected to occur at the potential gradients dealt with in Geiger counters, and other mechanisms, for instance the pulling apart of atoms in the gas, also seem to be out of the question because of the magnitude of the field which would be required.

Experiments I made some time ago seemed to substantiate the idea that high voltage alone cannot initiate the discharge. After allowing a counter to remain at its plateau or below for a few minutes, the voltage was suddenly raised to several hundred volts above the plateau. The first count after raising the voltage occurred substantially no sooner than it would have occurred under normal operation on the plateau. After the first count, further discharges came in rapid succession, as is normally observed above the plateau.

Lately I have carried the experiment somewhat further. By means of a system of relays, timers, etc., a counter was put through the following cycle: voltage raised to some high value (above plateau); time measured to first discharge; voltage dropped back to one-half the high value immediately after first discharge; resting period allowed at half-voltage; voltage again raised to high value. Precautions were taken to prevent any surges produced by the relay contacts from reaching the counter. The apparatus ran automatically, and at least 300 cycles were recorded at each setting of the time and voltage. The average time between the raising of the voltage and the first discharge is plotted in the two graphs (see Fig. 1); the first showing the time as a function of voltage with constant resting period and the second showing the time as a function of resting period with constant voltage. Data on the counter tube used are: wire, 10-mil tungsten; cathode, brass $\frac{3}{4}$-inch diameter; gas, 9-cm argon, 1-cm alcohol vapor. A

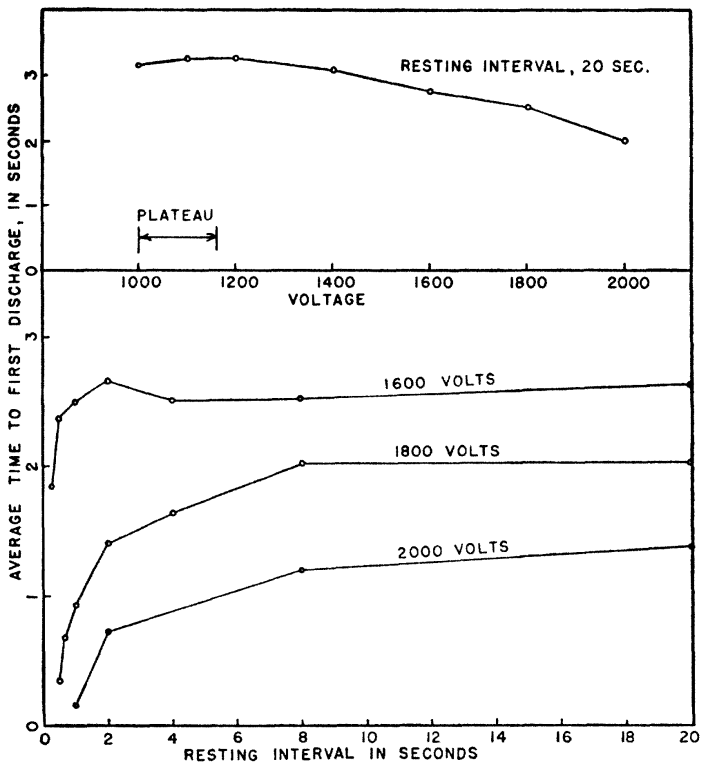

Fig. 1. Average time between raising of the voltage and the first discharge of a Geiger counter. Upper curve for constant resting period; lowe curve for different resting periods and various constant voltages. Correction 1600,2000 , and 2300 volts, part of the figure should have been labeled

Neher-Harper quench circuit was used. The plateau extended from 1000 to 1160 volts. The counter was housed in 2 inches of lead and was subject only to natural radiation background, which gave 20 counts per minute. This agreed with the average time for firing found with the relay scheme, when the voltage applied was within the plateau range.

The results make it reasonable to suppose that in the region above the plateau, spurious discharges may be initiated by an after-emission of electrons or photons, due to activation of the surfaces or gas produced by the previous discharge, rather than by ionization produced directly by the application of the high voltage. Since the curves show that in some cases it requires seconds for the after-emission to die away, it does not seem possible to account for the effect on the basis simply of gas ions which fail to be collected during the resting period. Activation of the metal or gas would be expected to depend strongly upon the kinds of materials present and to increase with increasing intensity of discharge. It appears to decay in a way similar to radioactive decay, as is indicated by the second set of curves. It would not be sensible, however, to try to derive the decay law of the after-emission from the curves because of the complexity of materials in the counter.

It should not be assumed that meager results presented here are representative of all counters, since only one example was used, but the measurements do indicate a line of investigation which well might lead to methods of increasing the width of the Geiger counter plateau.

I am grateful to Professors W. C. Parkinson, M. L. Wiedenbeck, R. W. Pidd, and L. Madansky for helpful discussions.

\section{High Multiplication Proportional Counters for Energy Measurements}

G. C. Hanna, D. H. W. Kirkwood, and B. Pontecorvo

Chalk River Laboratory, National Research Council of Canada, Chalk River, Ontario, Canado January 24, 1949

$\mathrm{D}$ EVELOPMENTS have been reported recently in the application of proportional counters to the detection ${ }^{1}$ of electrons and to the measurement ${ }^{2}$ of electron or $\beta$-ray 